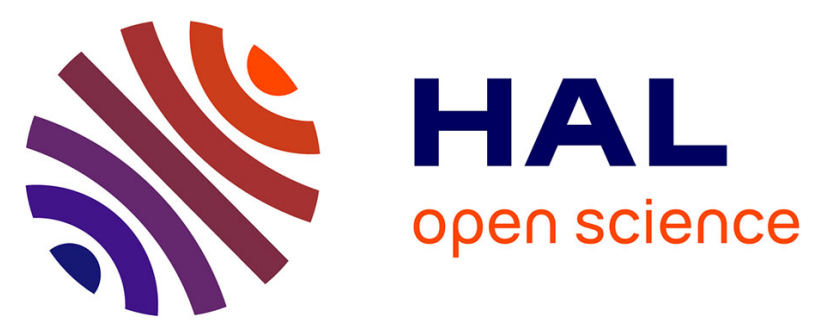

\title{
Effects of genetic entry and competition by neighbouring trees on growth and wood properties of cloned Norway spruce (Picea abies)
}

Ane Zubizarreta Gerendiain, Heli Peltola, Pertti Pulkkinen, Seppo Kellomäki

\section{- To cite this version:}

Ane Zubizarreta Gerendiain, Heli Peltola, Pertti Pulkkinen, Seppo Kellomäki. Effects of genetic entry and competition by neighbouring trees on growth and wood properties of cloned Norway spruce (Picea abies). Annals of Forest Science, 2009, 66 (8), 10.1051/forest/2009075 . hal-00883555

\section{HAL Id: hal-00883555 https://hal.science/hal-00883555}

Submitted on 1 Jan 2009

HAL is a multi-disciplinary open access archive for the deposit and dissemination of scientific research documents, whether they are published or not. The documents may come from teaching and research institutions in France or abroad, or from public or private research centers.
L'archive ouverte pluridisciplinaire HAL, est destinée au dépôt et à la diffusion de documents scientifiques de niveau recherche, publiés ou non, émanant des établissements d'enseignement et de recherche français ou étrangers, des laboratoires publics ou privés. 


\title{
Effects of genetic entry and competition by neighbouring trees on growth and wood properties of cloned Norway spruce (Picea abies)
}

\author{
Ane Zubizarreta Gerendiain ${ }^{1 *}$, Heli Peltola ${ }^{1}$, Pertti PulkKineN ${ }^{2}$, Seppo KellomäKi $^{1}$ \\ ${ }^{1}$ University of Joensuu, Faculty of Forest Sciences, P.O. Box 111, 80101 Joensuu, Finland \\ ${ }^{2}$ Finnish Forest Research Institute, Haapastensyrjä Breeding Station, 16200 Läyliäinen, Finland
}

(Received 3 December 2008; revised version 28 February 2009; accepted 10 March 2009)

Keywords:

growth /

wood density /

fibre property /

competition index /

provenance

\begin{abstract}
- The aim of this work was to study both the effects of genetic entry and competition by neighbouring trees on growth, yield, wood density traits and fibre properties of 20 Norway spruce clones grown in an experimental trial located in southern Finland. The material included 10 Finnish clones, 2 Russian clones and 8 provenance-hybrids clones, the latter ones representing crosses between Finnish and foreign parent trees.

- We found that, compared to growth and yield, wood density traits and fibre properties showed, on average, lower phenotypic variations. Moreover, significant differences could be observed among the clones regardless of the trait $(p<0.05)$. Conversely, on average, no clear differences could be found between Finnish, Russian and provenance-hybrids clones in most of the studied traits.

- The marked differences among the clones, and lack of clear differences among the provenances suggested that any ranking, regarding different traits, should be based on individual clones. The phenotypic correlations between different traits ranged from moderate to strong suggesting that selection based on one trait would affect other traits. Regardless of clone, the growth of trees (e.g. breast height diameter) decreased and the wood density increased with increasing competition by neighbouring trees, which was expressed by competition index.
\end{abstract}

Résumé - Effets de la part génétique et de la concurrence des arbres voisins sur la croissance et les propriétés du bois de clones d'épicéa (Picea abies).

- Le but de ce travail était d'étudier à la fois les effets de la part génétique et de la concurrence des arbres voisins sur la croissance des arbres, la production, les caractéristiques de la densité du bois et les propriétés des fibres de 20 clones d'épicéa cultivés dans un dispositif expérimental d'essai situé dans le sud de la Finlande. Le matériel était constitué de 10 clones finlandais, 2 clones de Russie et de 8 provenances de clones hybrides, ces derniers représentant les croisements entre arbres parents le Finlandais et étrangers.

- Nous avons trouvé que, comparativement à la croissance et à la production, la densité du bois et les caractéristiques des propriétés des fibres montraient, en moyenne, de plus faibles variations phénotypiques. En outre, des différences significatives ont pu être observées chez les clones, quel que soit le caractère $(p<0,05)$. Inversement, en moyenne, pour la plupart des caractéristiques étudiées, on n'a pas constaté de différences claires entre les clones finlandais, russes et les provenances de clones hybrides.

- Les différences marquées entre les clones, et l'absence de différences évidentes entre les provenances ont suggéré que tout classement, en ce qui concerne les différentes caractéristiques, devrait être basé sur des clones individuels. Les corrélations phénotypiques entre les différentes caractéristiques varient de modéré à fort, suggérant que la sélection sur la base d'une caractéristique serait de nature à affecter les autres caractéristiques. Indépendamment des clones, la croissance des arbres (par exemple le diamètre à hauteur de poitrine) a diminué et la densité du bois a augmenté avec l'accroissement de la concurrence des arbres voisins, qui a été exprimée par l'index de concurrence.

\footnotetext{
*Corresponding author: ane.zubizarreta@joensuu.fi
} 


\section{INTRODUCTION}

In general, it has been observed that any significant increase in the growth rate of coniferous species, such as Norway spruce (Picea abies (L.) H. Karst.) will increase earlywood percentage while at the same time decrease wood density and fibre length (Ekensted et al., 2003; Mäkinen et al., $2002 b)$. In this sense, the different growth and wood properties correlate with each other. Moreover, wood density affects fibre yield and fibre morphology, which are two of the main determinants of the final quantity and quality of paper products (Tyrväinen, 1995). Therefore, in addition to the quantity of raw material, sufficient information regarding its properties and relations would be important from the point of view of forest industry. If sufficient detailed information on raw material properties would be available it would facilitate its optimal use by the industry.

Such information would provide detailed understanding of how the growth and wood properties are affected by environmental conditions and controlled by silvicultural measures. Nonetheless, different genetic entries and/or provenances may show different properties even if grown in same conditions, as was observed, for example, among provenances in loblolly pine (Pinus taeda), Scots pine (Pinus sylvestris) and Norway spruce (Jayawickrama et al., 1997; Skrøppa et al., 1999; Ståhl, 1998). For instance, usually the northern provenances have the largest proportion of latewood and thus, higher wood density (Persson and Persson, 1997; Skrøppa and Magnussen, 1993), but there are contradictory results in this respect (Wilhemsson et al., 2002).

On the other hand, genetic entries with, for example, a high growth rate with relatively high overall wood density could be found, as was observed previously in black spruce (Picea mariana), lodgepole pine (Pinus contorta) and Norway spruce (Wang et al., 2000; Zhang and Morgenstern, 1995; Zubizarreta Gerendiain et al., 2007). This demonstrates that breeding may provide the opportunity to recognise genetic entries and/or provenances with an advantageous combination of growth and wood properties, for example.

Competition for resources (e.g. nutrients, water and light) directly affects the growth processes and crown development of individual trees within a stand as well as their allocation of growth and wood properties along the stem (Zobel and van Buijtenen, 1989). Therefore, it would be important to determine if any genetic entries could simultaneously maintain high growth rate as well as desired wood properties after canopy closure. In previous studies, competition indices (CI) have been used to measure the individual tree competition (e.g. Pukkala, 1989; Schütz, 1989). Their use is generally based on the assumption that a tree's competitive ability can be indicated by an expression of the number of competitors, their size and distance and/or spatial distribution of neighbouring trees, for example.

Nowadays, CIs are generally classified into two groups: (i) the distance independent indices, in which the main variables are related to the capacity of the target tree to grow and; (ii) the distance dependent indices, which measure the development of the target tree as a function of the characteristics and location of the neighbour trees (Tomé and Burkhart, 1989). So far, neither type of indices have been found to be superior in young regularly spaced plantations before canopy closure (e.g. Biging and Dobbertin, 1992; Mäkinen, 1997). On the other hand, recently some indices have included crown dimensions to incorporate the competition for light, with some success, for example for tree species such as Ponderosa pine (Pinus ponderosa) and Douglas-fir (Pseudotsuga menziesii) (Biging and Dobbertin, 1992).

In the above context, the aim of this work was to study both the effects of genetic entry and competition by neighbouring trees on growth and yield, wood density traits and fibre properties of 20 Norway spruce clones grown in an experimental trial located in southern Finland. In this context, we investigated if differences exist in different traits between Finnish and Russian clones, in addition to provenance-hybrids clones. The latter ones represented crosses between Finnish and foreign parent trees.

\section{MATERIAL AND METHODS}

\subsection{Experimental data}

The material for the present study was harvested from a unique Norway spruce clonal field trial, which was established in 1979 by the Finnish Forest Research Institute (test 690/01) in Kangasniemi, southern Finland $\left(61^{\circ} 59^{\prime} 3\right.$ N, $\left.26^{\circ} 38^{\prime} 54 \mathrm{E}\right)$. The trial was situated on a medium fertile forest site, Myrtillus type (Cajander, 1926), with a spacing of $2 \mathrm{~m} \times 2 \mathrm{~m}$. No thinning had been carried out up to the time of harvesting and canopy closure was reached at that time.

In November 2007, materials representing 20 Norway spruce clones were harvested. These 20 clones represented a total of 7 provenance or provenance-hybrids. Altogether, 10 of these 20 clones originated from Finnish plus trees $(\mathrm{F} \times \mathrm{F})$, representing different breeding regions in Finland. A further 2 clones originated from Russian parent trees $(R \times R)$. The remaining harvested clones (8) represented provenance-hybrids, with the parent being crosses between Finnish trees and either German (4 clones, $\mathrm{F} \times \mathrm{G})$, Swiss $(1, \mathrm{~F} \times \mathrm{S})$, Latvian (1, $\mathrm{F} \times \mathrm{L})$, Ukrainian $(1, \mathrm{~F} \times \mathrm{U})$ or Estonian $(\mathrm{F} \times \mathrm{E})$ parent trees (Tab. I). Both, the provenances and provenance-hybrids will be subsequently referred to as origin.

Five randomly selected samples were harvested for each clone (i.e. one tree per clone and blocks) and their height and diameter at breast height and at $6 \mathrm{~m}$ height were measured (Tab. I). Additionally, the crown size of the sample tree was measured based on the orthogonal projection, first in north-south direction and then in west-east direction. After felling the sample trees, sample discs were taken at a stem height of 1-1.3 $\mathrm{m}$ for laboratory analyses. Furthermore, breast height diameter, tree height and crown size for the eight trees surrounding the sample tree (in a square plot) were measured (i.e. sample tree was located in the middle).

\subsection{Laboratory measurements}

An ITRAX X-ray microdensitometer (Cox Analytical Systems, Göteborg, Sweden) was used to measure the intra-ring density profiles from pith to bark (for further details see Zubizarreta Gerendiain et al., 2007). A wood specimen of $5 \mathrm{~mm} \times 5 \mathrm{~mm}$ (i.e. a radial segment 
Table I. Harvested Norway spruce clones and the geographical origin of their mother and father trees.

\begin{tabular}{|c|c|c|c|c|c|c|}
\hline \multirow[b]{2}{*}{ Clone } & \multirow[b]{2}{*}{ Mother } & \multirow[b]{2}{*}{ Father } & \multicolumn{2}{|c|}{ Origin } & \multirow[t]{2}{*}{ Short name } & \multirow[t]{2}{*}{ Sample trees } \\
\hline & & & Mother & Father & & \\
\hline F48 & 44 Miehikkälä & $\mathrm{F}^{*}$ & Finland & Finland & $\mathrm{F} \times \mathrm{F}$ & 5 \\
\hline F331 & E2937/3 Pöytyä & $\mathrm{F}^{*}$ & Finland & Finland & $\mathrm{F} \times \mathrm{F}$ & 5 \\
\hline F334 & E2937/6 Pöytyä & $\mathrm{F}^{*}$ & Finland & Finland & $\mathrm{F} \times \mathrm{F}$ & 5 \\
\hline F337 & E2061/9 Jokioinen & $\mathrm{F}^{*}$ & Finland & Finland & $\mathrm{F} \times \mathrm{F}$ & 5 \\
\hline F338 & E2061/10 Jokioinen & $\mathrm{F}^{*}$ & Finland & Finland & $\mathrm{F} \times \mathrm{F}$ & 5 \\
\hline F345 & E2666/6 Loppi & $\mathrm{F}^{*}$ & Finland & Finland & $\mathrm{F} \times \mathrm{F}$ & 5 \\
\hline F354 & E2673/5 Loppi & $\mathrm{F}^{*}$ & Finland & Finland & $\mathrm{F} \times \mathrm{F}$ & 5 \\
\hline F311 & H5584 Loppi & $\mathrm{F}^{*}$ & Finland & Finland & $\mathrm{F} \times \mathrm{F}$ & 5 \\
\hline F374 & K1399 Pieksänmaa & K1398/10 Pieksänmaa & Finland & Finland & $\mathrm{F} \times \mathrm{F}$ & 5 \\
\hline $\mathrm{F} 430$ & K1399 Pieksänmaa & K1398/5 Pieksänmaa & Finland & Finland & $\mathrm{F} \times \mathrm{F}$ & 5 \\
\hline R325 & 513/2 Pskov & $\mathrm{F}^{*}$ & Russia & Russia & $\mathrm{R} \times \mathrm{R}$ & 4 \\
\hline R330 & 517/1 Novgorod & $\mathrm{F}^{*}$ & Russia & Russia & $\mathrm{R} \times \mathrm{R}$ & 5 \\
\hline S371 & E2705 Muurame & E1487/1 Wintshgau & Finland & Switzerland & $\mathrm{F} \times \mathrm{S}$ & 5 \\
\hline L373 & E2654 Loppi & E941 Goldingen & Finland & Latvia & $\mathrm{F} \times \mathrm{L}$ & 5 \\
\hline E386 & E1240 Pieksänmaa & E949/1 Perewald & Finland & Estonia & $\mathrm{F} \times \mathrm{E}$ & 5 \\
\hline U3030 & E2089 Längelmäki & E4337 Volovec & Finland & Ukraine & $\mathrm{F} \times \mathrm{U}$ & 5 \\
\hline G380 & E268 Lohja & E1485/4 Schielbach & Finland & Germany & $\mathrm{F} \times \mathrm{G}$ & 5 \\
\hline G384 & E456D Iitti & E1485 Spiegelau & Finland & Germany & $\mathrm{F} \times \mathrm{G}$ & 5 \\
\hline G477 & H3499 Loppi & E4277 Schmiedewald & Finland & Germany & $\mathrm{F} \times \mathrm{G}$ & 5 \\
\hline G478 & E5580 Loppi & E1893 Garlsfeld & Finland & Germany & $\mathrm{F} \times \mathrm{G}$ & 5 \\
\hline
\end{tabular}

$\mathrm{F}^{*}$, open pollination.

from pith to bark from the southern side of the tree) was cut from each sample disc and kept under controlled conditions to achieve a moisture content of $12 \%$ (air dry). The X-ray radiographic images were thereafter analysed with the Density software (Bergsten et al., 2001) and excel macros, based on which we determined the following variables for each annual ring: ring width ( $\mathrm{RW}, \mathrm{mm}$ ), earlywood and latewood widths (EWW; LWW, mm), earlywood and latewood densities and mean ring density (EWD; LWD; WD, $\mathrm{g} / \mathrm{cm}^{3}$ ). The mean of the maximum and minimum intraring densities were used as the threshold for earlywood and latewood in each ring, as was done in previous corresponding works by Zubizarreta Gerendiain et al. (2007).

Thereafter, small specimens of wood, each representing two annual rings from pith to bark, were chipped away from the same sample discs that were used for X-ray analyses. The specimens were subsequently macerated in a boiling $1: 1(\mathrm{v} / \mathrm{v})$ mixture of acetic acid and hydrogen peroxide. An L\&W Fiber Tester (AB Lorentzen \& Wettre, Kista, Sweden) was used for intra-ring analysis of fibre properties, automatically providing averages of fibre length (FL) and fibre width (FW) for each specimen. Additionally, average fibre coarseness (C, fibre mass per unit length) and fibre wall thickness (FWT) could be calculated based on these measurements (see details for methodology in Zubizarreta Gerendiain et al., 2008).

\subsection{Data analyses}

Stem volume $\left(V, \mathrm{~m}^{3}\right)$ was calculated for each sample tree based on tree height and stem diameters at 1.3 and $6 \mathrm{~m}$ (see Laasasenaho, 1982). Intra-ring properties, measured for each ring or ring pairs from pith to bark, were used to calculate the weighted averages for different wood density traits and fibre properties for each sample tree. This was done by weighting each ring value with its corresponding ring width (see Zubizarreta Gerendiain et al., 2007). Additionally, the means for RW, EWW, LWW and latewood proportion (LWW\%) were determined for each sample tree.

The phenotypic coefficient of variation $(C V)$ was calculated by normalising the standard deviation $(\sigma)$ with the mean $(\mu)$ of each trait and clone (i.e. $C V=\sigma \times 100 / \mu$ ). Furthermore, phenotypic correlations $\left(r_{\mathrm{p}}\right)$ between different traits were computed using the Pearson's correlation method. To identify if differences existed, on average, in the studied traits between Finnish, Russian or provenancehybrids clones (i.e. crosses between Finnish and foreign parent trees) we grouped the 20 clones into 7 different origins. Thereafter, analysis of variance was performed for growth, yield, wood density traits and fibre properties using the General Linear Model (GLM) procedure (SPSS for Windows, 15.0, Chicago, IL). The effect of origin was first tested, followed by the effect of clone, using, in both cases, the effect of the block as a random factor (Eq. (1))

$$
Y_{i j k}=\mu+C_{i}+B_{j}+e_{i j k}
$$

where $Y_{i j k}$ is the studied trait, $\mu$ is the general mean, $C_{i}$ is the clone or origin fixed effect, $B_{j}$ is the random effect of the block, and $e_{i j k}$ is the residual effect.

To study the effects of competition by neighbouring trees on different traits, we used a competition index (CI) formulated by Schütz (1989, see Eq. (2)). This CI is calculated based on crown characteristics and tree size, and thus, also reveals the competition for light at canopy closure unlike other CIs considered (e.g. Hegyi, 1984). In the follow up analyses CI was included as a covariate in the GLM, where clone was used as a fixed factor and block as a random factor. The interaction between clone and CI was also tested, but not included in the models, since it was not statistically significant. We analysed different 
Table II. Average statistics and phenotypic coefficient of variation $(\mathrm{CV}, \%)$ for breast height diameter $(\mathrm{cm})$, height $(\mathrm{m})$, stem volume $\left(\mathrm{m}^{3}\right)$, ring width (RW, mm) and latewood width \% (LWW\%) for the 20 clones.

\begin{tabular}{|c|c|c|c|c|c|c|c|c|c|c|c|}
\hline \multirow{2}{*}{ Origin } & \multirow{2}{*}{ Clone } & \multicolumn{2}{|c|}{ Diameter } & \multicolumn{2}{|c|}{ Height } & \multicolumn{2}{|c|}{ Volume } & \multicolumn{2}{|l|}{ RW } & \multicolumn{2}{|c|}{ LWW\% } \\
\hline & & Mean $\pm \mathrm{sd}$ & $\mathrm{CV}$ & Mean \pm sd & $\mathrm{CV}$ & Mean \pm sd & $\mathrm{CV}$ & Mean \pm sd & $\mathrm{CV}$ & Mean \pm sd & $\mathrm{CV}$ \\
\hline \multirow{10}{*}{$\mathrm{F} \times \mathrm{F}$} & F48 & $11.7 \pm 5.3$ & 45 & $11.5 \pm 4.1$ & 36 & $0.082 \pm 0.073$ & 88 & $2.30 \pm 0.87$ & 38 & $27.2 \pm 5.3$ & 20 \\
\hline & F331 & $12.6 \pm 5.0$ & 40 & $11.4 \pm 2.8$ & 25 & $0.080 \pm 0.057$ & 72 & $2.49 \pm 0.79$ & 32 & $23.0 \pm 3.5$ & 15 \\
\hline & F311 & $9.0 \pm 2.3$ & 25 & $10.1 \pm 2.2$ & 21 & $0.038 \pm 0.020$ & 52 & $1.84 \pm 0.37$ & 20 & $30.9 \pm 6.8$ & 22 \\
\hline & F334 & $12.3 \pm 1.6$ & 13 & $12.1 \pm 1.1$ & 9 & $0.074 \pm 0.023$ & 31 & $2.57 \pm 0.37$ & 14 & $25.2 \pm 1.0$ & 4 \\
\hline & F337 & $8.0 \pm 2.1$ & 26 & $8.1 \pm 3.0$ & 37 & $0.027 \pm 0.021$ & 77 & $1.84 \pm 0.48$ & 26 & $29.4 \pm 7.3$ & 25 \\
\hline & F338 & $13.2 \pm 1.5$ & 11 & $13.1 \pm 1.1$ & 8 & $0.091 \pm 0.017$ & 19 & $2.74 \pm 0.50$ & 18 & $21.8 \pm 2.9$ & 13 \\
\hline & F345 & $9.3 \pm 4.6$ & 50 & $8.5 \pm 3.3$ & 39 & $0.042 \pm 0.034$ & 80 & $2.31 \pm 1.12$ & 48 & $26.9 \pm 8.3$ & 31 \\
\hline & F354 & $13.8 \pm 2.0$ & 14 & $11.9 \pm 0.5$ & 4 & $0.093 \pm 0.023$ & 25 & $2.86 \pm 0.71$ & 25 & $26.1 \pm 4.8$ & 18 \\
\hline & F374 & $13.1 \pm 1.5$ & 11 & $11.6 \pm 1.7$ & 14 & $0.079 \pm 0.027$ & 34 & $2.77 \pm 0.46$ & 17 & $26.1 \pm 4.5$ & 17 \\
\hline & F430 & $14.9 \pm 3.8$ & 26 & $12.6 \pm 1.3$ & 10 & $0.114 \pm 0.057$ & 50 & $3.13 \pm 0.86$ & 27 & $22.1 \pm 4.5$ & 20 \\
\hline \multirow{2}{*}{$\mathrm{R} \times \mathrm{R}$} & R325 & $10.2 \pm 4.9$ & 48 & $10.1 \pm 2.7$ & 27 & $0.056 \pm 0.066$ & 119 & $2.00 \pm 0.45$ & 23 & $29.3 \pm 5.0$ & 17 \\
\hline & R330 & $9.7 \pm 4.3$ & 45 & $9.2 \pm 3.4$ & 37 & $0.045 \pm 0.042$ & 93 & $2.17 \pm 0.73$ & 34 & $34.1 \pm 10.7$ & 31 \\
\hline $\mathrm{F} \times \mathrm{S}$ & S371 & $9.9 \pm 2.9$ & 29 & $11.5 \pm 2.2$ & 19 & $0.051 \pm 0.034$ & 67 & $2.10 \pm 0.64$ & 30 & $25.3 \pm 2.5$ & 10 \\
\hline $\mathrm{F} \times \mathrm{L}$ & L373 & $10.4 \pm 2.4$ & 24 & $10.0 \pm 1.8$ & 18 & $0.048 \pm 0.028$ & 57 & $2.60 \pm 0.54$ & 21 & $26.5 \pm 2.7$ & 10 \\
\hline \multirow{4}{*}{$\mathrm{F} \times \mathrm{G}$} & G380 & $10.9 \pm 3.8$ & 34 & $11.1 \pm 3.9$ & 35 & $0.069 \pm 0.049$ & 71 & $2.41 \pm 0.49$ & 20 & $26.9 \pm 6.4$ & 23 \\
\hline & G384 & $7.7 \pm 3.1$ & 41 & $6.8 \pm 2.7$ & 40 & $0.022 \pm 0.018$ & 81 & $2.19 \pm 0.32$ & 15 & $24.0 \pm 2.5$ & 10 \\
\hline & G477 & $13.9 \pm 4.8$ & 35 & $11.7 \pm 2.1$ & 18 & $0.100 \pm 0.057$ & 57 & $3.08 \pm 0.94$ & 31 & $22.9 \pm 4.2$ & 18 \\
\hline & G478 & $11.1 \pm 2.4$ & 22 & $10.3 \pm 1.7$ & 17 & $0.057 \pm 0.028$ & 49 & $2.72 \pm 0.57$ & 21 & $23.4 \pm 3.2$ & 14 \\
\hline $\mathrm{F} \times \mathrm{E}$ & E386 & $10.3 \pm 4.3$ & 42 & $10.4 \pm 4.9$ & 47 & $0.058 \pm 0.040$ & 69 & $2.18 \pm 0.66$ & 30 & $26.0 \pm 3.8$ & 15 \\
\hline $\mathrm{F} \times \mathrm{U}$ & U3030 & $11.2 \pm 1.9$ & 17 & $10.3 \pm 1.7$ & 17 & $0.056 \pm 0.020$ & 36 & $2.57 \pm 0.64$ & 25 & $21.7 \pm 4.1$ & 19 \\
\hline \multicolumn{2}{|c|}{ Mean } & $11.1 \pm 2.0$ & 18 & $10.6 \pm 1.6$ & 15 & $0.064 \pm 0.025$ & 39 & $2.44 \pm 0.38$ & 15 & $25.9 \pm 3.2$ & 12 \\
\hline
\end{tabular}

responses between clones for tree height, breast height diameter, RW, and WD, which we expected to be most affected by the competition.

$$
C I_{\text {Schütz }}=\sum_{i=1}^{n} 0.5-\frac{d_{i j}-\left(c r_{j}+c r_{i}\right)}{\left(c r_{j}+c r_{i}\right)}+0.65 \frac{h_{i}-h_{j}}{d_{i j}}
$$

where $j$ is the competitor tree and $i$ the sample tree, $n$ is the number of neighbours, $d_{i j}(\mathrm{~m})$ is the horizontal distance between the target tree and competitor, $h(\mathrm{~m})$ is tree's height and $c r(\mathrm{~m})$ is the mean crown radius. In this approach, a neighbouring tree is only included if the calculated value is greater than zero.

\section{RESULTS}

\subsection{Variation among clones and origins}

Among the yield traits, height $(H)$ showed, on average, the smallest phenotypic variation $(C V)$ (average of 15\%), followed by diameter $(D, 18 \%)$ and stem volume $(V, 39 \%)$ (Tab. II). In addition, all the growth traits showed a similar phenotypic variation compared to $D$ and $H$, but much lower than $V$ (Tab. II). Among the individual clones, significant differences for all growth and yield traits was found $(p<0.05)$ (Tab. III). In this respect, clone F430 $(\mathrm{F} \times \mathrm{F})$ had the largest $D$ and $V$, as well as the second largest $H$, being $33 \%, 78 \%$ and $19 \%$ larger than the averages over all the clones, respectively. On the contrary, clone $\mathrm{G} 384(\mathrm{~F} \times \mathrm{G})$ showed the smallest $V, H$ and $D$, being $35 \%, 64 \%$ and $69 \%$ of the average for all the clones, respectively.

In comparison with growth and yield traits, wood density traits showed lower phenotypic variation. Overall wood density (WD) had, on average, the larger phenotypic variation
Table III. Analysis of variance $\left(F\right.$-value and correspondent $\left.\mathrm{p}^{1}\right)$ for clonal effects or origin effect on diameter at breast height, height, stem volume, earlywood and latewood width (EWW, LWW), latewood width \% (LWW\%), ring width (RW), earlywood, latewood and overall wood density (EWD, LWD, WD), fibre length and width (FL, FW), coarseness (C) and fibre wall thickness (FWT).

\begin{tabular}{lcccccc}
\hline \multirow{2}{*}{ Trait } & \multicolumn{3}{c}{ Statistic for Clones } & \multicolumn{3}{c}{ Statistic for Origins } \\
\cline { 2 - 7 } & MSE & F-ratio & $P$-value & MSE & F-ratio & $P$-value \\
\hline Diameter & 10.70 & 1.874 & $\mathbf{0 . 0 3}$ & 12.85 & 0.68 & 0.67 \\
Height & 7.014 & 1.772 & $\mathbf{0 . 0 4}$ & 8.241 & 0.75 & 0.61 \\
Volume & 0.001 & 2.190 & $\mathbf{0 . 0 1}$ & 0.002 & 0.77 & 0.60 \\
EWW & 0.235 & 2.361 & $\mathbf{0 . 0 0}$ & 0.287 & 1.71 & 0.13 \\
LWW & 0.011 & 2.072 & $\mathbf{0 . 0 1}$ & 0.013 & 1.34 & 0.25 \\
LWW\% & 25.39 & 2.056 & $\mathbf{0 . 0 1}$ & 27.22 & 3.08 & $\mathbf{0 . 0 1}$ \\
RW & 0.302 & 2.324 & $\mathbf{0 . 0 1}$ & 0.374 & 1.42 & 0.22 \\
EWD & 0.000 & 4.516 & $\mathbf{0 . 0 0}$ & 0.000 & 1.81 & 0.11 \\
LWD & 0.001 & 3.600 & $\mathbf{0 . 0 0}$ & 0.001 & 4.25 & $\mathbf{0 . 0 0}$ \\
WD & 0.001 & 3.785 & $\mathbf{0 . 0 0}$ & 0.001 & 3.07 & $\mathbf{0 . 0 1}$ \\
FL & 0.037 & 1.871 & $\mathbf{0 . 0 3}$ & 0.042 & 1.49 & 0.19 \\
FW & 2.861 & 1.924 & $\mathbf{0 . 0 2}$ & 3.272 & 1.61 & 0.15 \\
FWT & 0.007 & 1.985 & $\mathbf{0 . 0 2}$ & 0.007 & $\mathbf{2 . 4 5}$ & $\mathbf{0 . 0 3}$ \\
C & 318.5 & 1.660 & 0.06 & 339.3 & 2.01 & 0.07 \\
\hline
\end{tabular}

${ }^{1}$ Significance of F-ratio with $p<0.05$ in bold.

(5.3\%) compared to earlywood (EWD) and latewood (LWD) densities (4.7 and 4.5\%, respectively) (Tab. IV). Moreover, the differences observed for all wood density traits, among the individual clones, were significant $(p<0.05)$ (Tab. III). For instance, clone $\mathrm{R} 330(\mathrm{R} \times \mathrm{R})$ showed the highest $\mathrm{WD}$, with $10 \%$ above the average for all the clones, while clone G384 $(\mathrm{F} \times \mathrm{G})$ 
Table IV. Average statistics and phenotypic coefficient of variation $(\mathrm{CV}, \%)$ for earlywood $\left(\mathrm{EWD}, \mathrm{g} / \mathrm{cm}^{3}\right)$, latewood $\left(\mathrm{LWD}, \mathrm{g} / \mathrm{cm}^{3}\right)$ and overall wood density $\left(\mathrm{WD}, \mathrm{g} / \mathrm{cm}^{3}\right.$ ) for the 20 clones.

\begin{tabular}{|c|c|c|c|c|c|c|c|}
\hline \multirow{2}{*}{ Origin } & \multirow{2}{*}{ Clone } & \multicolumn{2}{|c|}{ EWD } & \multicolumn{2}{|c|}{ LWD } & \multicolumn{2}{|c|}{ WD } \\
\hline & & Mean \pm sd & $\mathrm{CV}$ & Mean \pm sd & $\mathrm{CV}$ & Mean \pm sd & $\mathrm{CV}$ \\
\hline \multirow{10}{*}{$\mathrm{F} \times \mathrm{F}$} & F48 & $0.357 \pm 0.017$ & 4.8 & $0.617 \pm 0.034$ & 5.4 & $0.428 \pm 0.032$ & 7.5 \\
\hline & F331 & $0.331 \pm 0.027$ & 8.0 & $0.588 \pm 0.038$ & 6.5 & $0.390 \pm 0.037$ & 9.5 \\
\hline & F311 & $0.344 \pm 0.011$ & 3.2 & $0.618 \pm 0.031$ & 5.0 & $0.428 \pm 0.023$ & 5.4 \\
\hline & F334 & $0.351 \pm 0.013$ & 3.8 & $0.623 \pm 0.024$ & 3.8 & $0.419 \pm 0.016$ & 3.9 \\
\hline & F337 & $0.377 \pm 0.035$ & 9.2 & $0.623 \pm 0.040$ & 6.5 & $0.449 \pm 0.045$ & 10.1 \\
\hline & F338 & $0.361 \pm 0.006$ & 1.7 & $0.616 \pm 0.019$ & 3.1 & $0.417 \pm 0.015$ & 3.6 \\
\hline & F345 & $0.321 \pm 0.014$ & 4.2 & $0.568 \pm 0.026$ & 4.5 & $0.386 \pm 0.028$ & 7.3 \\
\hline & F354 & $0.334 \pm 0.022$ & 6.7 & $0.575 \pm 0.010$ & 1.8 & $0.396 \pm 0.028$ & 7.0 \\
\hline & F374 & $0.327 \pm 0.013$ & 3.9 & $0.605 \pm 0.027$ & 4.5 & $0.399 \pm 0.020$ & 5.0 \\
\hline & F430 & $0.327 \pm 0.019$ & 5.9 & $0.584 \pm 0.036$ & 6.2 & $0.384 \pm 0.032$ & 8.3 \\
\hline \multirow{2}{*}{$\mathrm{R} \times \mathrm{R}$} & R325 & $0.326 \pm 0.016$ & 5.0 & $0.588 \pm 0.037$ & 6.3 & $0.404 \pm 0.033$ & 8.1 \\
\hline & R330 & $0.363 \pm 0.024$ & 6.6 & $0.633 \pm 0.048$ & 7.6 & $0.452 \pm 0.038$ & 8.4 \\
\hline $\mathrm{F} \times \mathrm{S}$ & S371 & $0.334 \pm 0.012$ & 3.6 & $0.633 \pm 0.028$ & 4.4 & $0.410 \pm 0.016$ & 3.9 \\
\hline $\mathrm{F} \times \mathrm{L}$ & L373 & $0.339 \pm 0.005$ & 1.5 & $0.598 \pm 0.028$ & 4.6 & $0.408 \pm 0.015$ & 3.7 \\
\hline \multirow{4}{*}{$\mathrm{F} \times \mathrm{G}$} & G380 & $0.325 \pm 0.019$ & 6.0 & $0.564 \pm 0.017$ & 3.0 & $0.388 \pm 0.028$ & 7.3 \\
\hline & G384 & $0.320 \pm 0.024$ & 7.5 & $0.547 \pm 0.024$ & 4.4 & $0.375 \pm 0.018$ & 4.9 \\
\hline & G477 & $0.334 \pm 0.016$ & 4.7 & $0.543 \pm 0.040$ & 7.5 & $0.382 \pm 0.025$ & 6.6 \\
\hline & G478 & $0.343 \pm 0.013$ & 3.7 & $0.601 \pm 0.046$ & 7.7 & $0.403 \pm 0.023$ & 5.7 \\
\hline $\mathrm{F} \times \mathrm{E}$ & E386 & $0.361 \pm 0.009$ & 2.6 & $0.591 \pm 0.046$ & 7.7 & $0.422 \pm 0.016$ & 3.8 \\
\hline \multirow[t]{2}{*}{$\mathrm{F} \times \mathrm{U}$} & U3030 & $0.335 \pm 0.008$ & 2.4 & $0.604 \pm 0.014$ & 2.4 & $0.393 \pm 0.017$ & 4.4 \\
\hline & & $0.340 \pm 0.016$ & 4.7 & $0.596 \pm 0.027$ & 4.5 & $0.407 \pm 0.021$ & 5.3 \\
\hline
\end{tabular}

Table V. Average statistics and phenotypic coefficient of variation $(\mathrm{CV}, \%)$ for fibre length $(\mathrm{mm})$ and fibre width ( $\mu \mathrm{m})$, fibre wall thickness $(\mathrm{FWT}, \mu \mathrm{m})$ and coarseness $(\mu \mathrm{g} / \mathrm{m})$ for the 20 clones.

\begin{tabular}{|c|c|c|c|c|c|c|c|c|c|}
\hline \multirow{2}{*}{ Origin } & \multirow{2}{*}{ Clone } & \multicolumn{2}{|c|}{ Fibre length } & \multicolumn{2}{|c|}{ Fibre width } & \multicolumn{2}{|c|}{ Coarseness } & \multicolumn{2}{|c|}{ FWT } \\
\hline & & Mean $\pm \mathrm{sd}$ & $\mathrm{CV}$ & Mean \pm sd & $\mathrm{CV}$ & Mean \pm sd & $\mathrm{CV}$ & Mean $\pm \mathrm{sd}$ & $\mathrm{CV}$ \\
\hline \multirow{10}{*}{$\mathrm{F} \times \mathrm{F}$} & F48 & $1.82 \pm 0.15$ & 8.4 & $27.0 \pm 2.1$ & 7.9 & $152 \pm 16$ & 10.8 & $1.25 \pm 0.07$ & 5.4 \\
\hline & F331 & $2.07 \pm 0.10$ & 4.8 & $28.9 \pm 0.7$ & 2.3 & $165 \pm 5$ & 2.9 & $1.26 \pm 0.04$ & 3.3 \\
\hline & F311 & $2.03 \pm 0.10$ & 4.8 & $27.4 \pm 1.0$ & 3.8 & $161 \pm 9$ & 5.5 & $1.31 \pm 0.03$ & 2.0 \\
\hline & F334 & $1.95 \pm 0.07$ & 3.8 & $28.1 \pm 1.1$ & 3.8 & $153 \pm 5$ & 3.3 & $1.21 \pm 0.03$ & 2.8 \\
\hline & F337 & $1.86 \pm 0.21$ & 11.2 & $27.5 \pm 1.7$ & 6.1 & $152 \pm 15$ & 9.8 & $1.23 \pm 0.06$ & 4.8 \\
\hline & F338 & $1.93 \pm 0.15$ & 7.6 & $29.9 \pm 1.1$ & 3.8 & $163 \pm 11$ & 6.9 & $1.21 \pm 0.08$ & 7.0 \\
\hline & F345 & $1.89 \pm 0.29$ & 15.2 & $26.5 \pm 2.1$ & 7.9 & $139 \pm 17$ & 12.5 & $1.16 \pm 0.08$ & 6.9 \\
\hline & F354 & $1.84 \pm 0.11$ & 6.2 & $26.6 \pm 0.5$ & 1.8 & $140 \pm 5$ & 3.9 & $1.17 \pm 0.05$ & 4.3 \\
\hline & F374 & $2.02 \pm 0.05$ & 2.5 & $27.2 \pm 0.6$ & 2.1 & $151 \pm 11$ & 7.4 & $1.23 \pm 0.07$ & 5.8 \\
\hline & F430 & $1.99 \pm 0.14$ & 7.0 & $28.6 \pm 0.7$ & 2.3 & $164 \pm 13$ & 7.8 & $1.28 \pm 0.09$ & 7.0 \\
\hline \multirow{2}{*}{$\mathrm{R} \times \mathrm{R}$} & R325 & $2.07 \pm 0.10$ & 4.7 & $27.3 \pm 0.9$ & 3.2 & $155 \pm 10$ & 6.8 & $1.26 \pm 0.06$ & 4.5 \\
\hline & R330 & $1.97 \pm 0.26$ & 13.3 & $28.4 \pm 3.3$ & 11.7 & $167 \pm 36$ & 21.5 & $1.29 \pm 0.13$ & 9.7 \\
\hline $\mathrm{F} \times \mathrm{S}$ & S371 & $1.98 \pm 0.15$ & 7.7 & $27.7 \pm 1.7$ & 6.2 & $161 \pm 21$ & 12.8 & $1.29 \pm 0.10$ & 7.6 \\
\hline $\mathrm{F} \times \mathrm{L}$ & L373 & $1.88 \pm 0.11$ & 6.0 & $26.5 \pm 0.9$ & 3.3 & $144 \pm 7$ & 5.0 & $1.21 \pm 0.07$ & 5.5 \\
\hline \multirow{4}{*}{$\mathrm{F} \times \mathrm{G}$} & G380 & $2.03 \pm 0.17$ & 8.3 & $27.4 \pm 1.3$ & 4.8 & $153 \pm 19$ & 12.6 & $1.24 \pm 0.11$ & 9.1 \\
\hline & G384 & $1.69 \pm 0.29$ & 17.4 & $25.9 \pm 1.6$ & 6.3 & $131 \pm 23$ & 17.3 & $1.11 \pm 0.13$ & 11.7 \\
\hline & G477 & $1.77 \pm 0.22$ & 12.3 & $28.1 \pm 1.9$ & 6.6 & $149 \pm 20$ & 13.4 & $1.17 \pm 0.10$ & 8.5 \\
\hline & G478 & $2.10 \pm 0.15$ & 7.3 & $28.5 \pm 1.1$ & 3.8 & $159 \pm 17$ & 10.6 & $1.23 \pm 0.10$ & 7.8 \\
\hline $\mathrm{F} \times \mathrm{E}$ & E386 & $1.75 \pm 0.39$ & 22.5 & $27.8 \pm 3.7$ & 13.4 & $149 \pm 36$ & 24.2 & $1.17 \pm 0.16$ & 13.7 \\
\hline $\mathrm{F} \times \mathrm{U}$ & U3030 & $2.08 \pm 0.22$ & 10.5 & $29.9 \pm 1.4$ & 4.6 & $175 \pm 20$ & 11.6 & $1.30 \pm 0.11$ & 8.7 \\
\hline \multicolumn{2}{|c|}{ Mean } & $1.94 \pm 0.12$ & 6.2 & $27.8 \pm 1.1$ & 3.9 & $154 \pm 11$ & 6.9 & $1.23 \pm 0.05$ & 4.4 \\
\hline
\end{tabular}



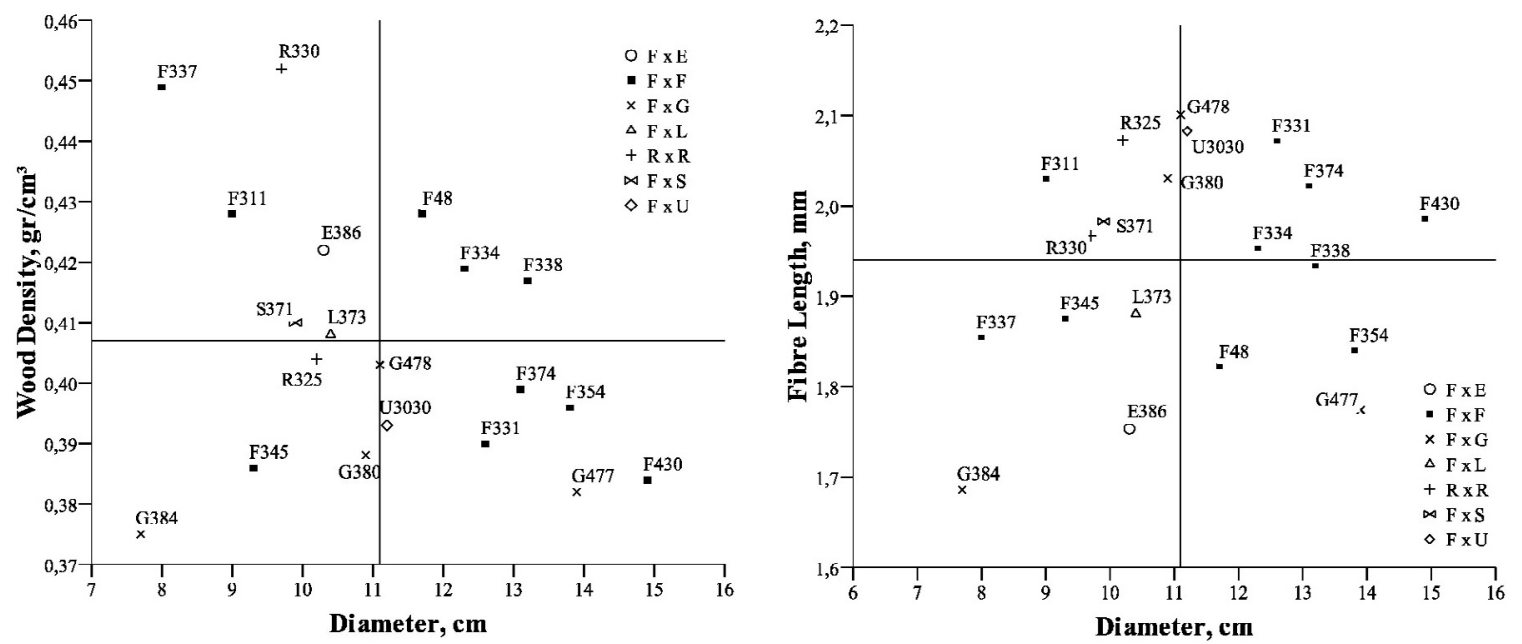

Figure 1. Relationships between breast height diameter, overall wood density and fibre length for 20 clones. The lines represent the average over all clones for each trait.

showed the lowest EWD and WD, being $94 \%$ and $92 \%$ of the average, respectively (Tab. IV).

Regarding fibre traits, fibre width (FW) and fibre wall thickness (FWT) had, on average, the lowest phenotypic variation (both around 4\%), followed by fibre length (FL) and coarseness (C) (6-7\%) (Tab. V). Among the individual clones, differences for FL and FW, in addition to FWT, were found $(p<0.05)$ (Tab. III). For instance, clone G478 (F×G) had the longest fibres (9\% above the average), while clone G384 had the shortest fibres (85\% of the average).

Some of the 20 clones showed both higher $D$ and WD than the average for all the clones, for example, clones F48, F334 and F338 (Fig. 1). Furthermore, F334 and F338 also had similar mean FL than the average for all the clones (Fig. 1), while having above average FW compared to the other clones. On the contrary, clone G384 showed the poorest growth, in addition to the lowest WD and FL (Fig. 1).

When grouping the clones into different origins, on average, Finnish origin $(\mathrm{F} \times \mathrm{F})$ had $10 \%$ larger $D$ and $24 \%$ higher $V$ than the average, while the Finnish-German $(F \times G)$ origin had the widest annual rings. However, no significant differences were found between origins for any of the yield traits $(D, H$ and $V)(p>0.05)$, while for growth traits only differences for LWW\% were significant (Tab. III) $(p<0.05)$. Regarding WD, the origins ranged from $0.43 \mathrm{~g} / \mathrm{cm}^{3}$ for Russian-Russian origin $(\mathrm{R} \times \mathrm{R})$ to $0.39 \mathrm{~g} / \mathrm{cm}^{3}$ for German-Finnish crosses $(\mathrm{F} \times \mathrm{G})$, with the difference being statistically significant $(p<0.05)$ (Tab. II). Similarly, observed differences between origins were significant for LWD, but not for EWD (Tab. III). On the other hand, when looking at fibre properties, the Finnish-Ukrainian $(\mathrm{F} \times \mathrm{U})$ origin had, on average, the longest and widest fibres (on average, FL $>2 \mathrm{~mm}$ and FW almost $30 \mu \mathrm{m}$ ). Nonetheless, the differences observed among the origins, in respect to fibre properties, were only significant for FWT $(p<0.05)$ (Tab. III).

\subsection{Effects of neighbours' competition on different traits}

Tree height $(H)$ and breast height diameter $(D)$ were affected by competition from neighbours, showing both a statistically significant negative correlation with the competition index (CI) over all the clones (for $D$ and $H,-0.59$ and -0.55 respectively, $p<0.05$ ) (Fig. 2) (CI is calculated based on $H$ ). Likewise, moderate negative correlation was observed between $\mathrm{CI}$ and ring width (RW) $(-0.56, p<0.05)$. In contrast, when the CI value increased, the overall wood density (WD) also increased (correlation of 0.33, $p<0.05$ ) (Fig. 2). By including the $\mathrm{CI}$ in the statistical analyses as a covariate for $D, H$ and $\mathrm{RW}$, the mean square error was also reduced (see Tabs. III and VI). Nonetheless, CI could not be used as a covariate to explain WD.

\subsection{Phenotypic correlations among different traits}

The phenotypic correlations among the different growth and yield traits were positive and moderate to high $(p<0.05)$. Similarly, the correlations among the different wood density traits were positive, ranging from moderate to strong ( $p<$ 0.05). In contrast, all the correlations between WD or EWD and different yield and wood property traits were negative $(p<0.05)$, from weak (e.g. between LWW and WD, -0.24$)$ to strong (between EWW and WD, -0.71). LWD showed both negative and positive correlations with the different growth and yield traits, but in most cases was statistically non significant $(p>0.05)$ (Tab. VII). The correlation observed among the different fibre properties were positive and statistically significant $(p<0.05)$, ranging from moderate (between FW and FWT, 0.55) to strong (between C and FWT, 0.89) (Tab. VII). In addition, FL, FW and $\mathrm{C}$ showed positive correlations with all yield traits, as well as with $\operatorname{LWD}(p<0.05)$. 

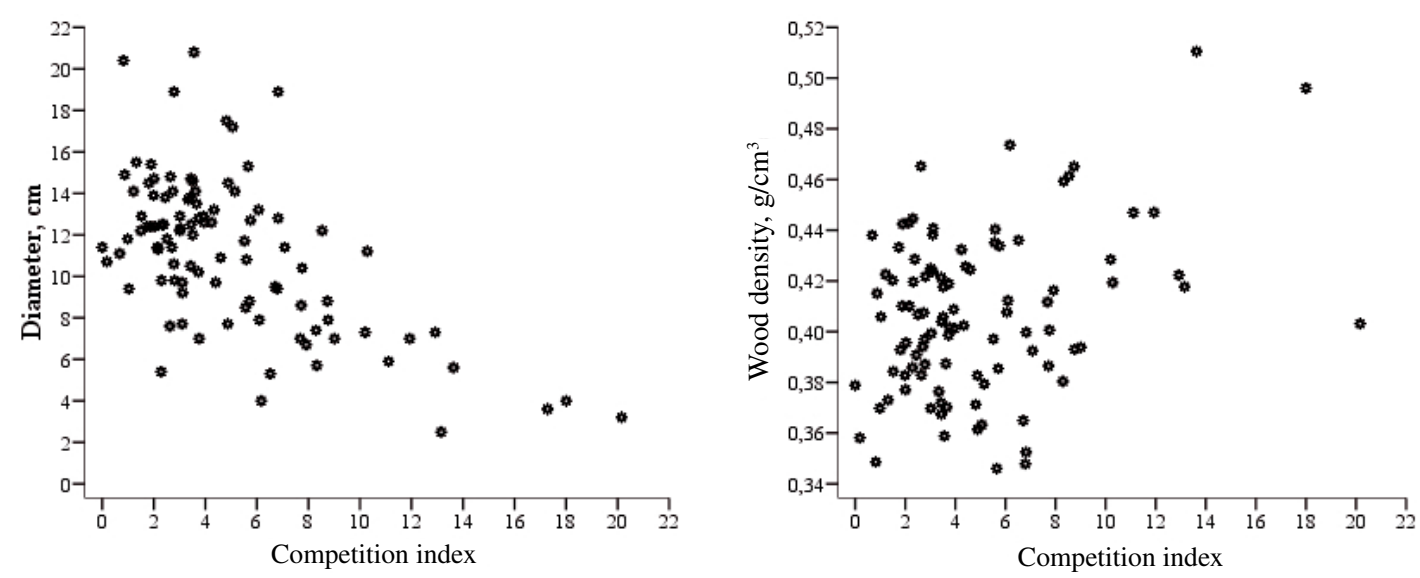

Figure 2. Relations between competition index (Schutz, 1989) and breast height diameter and overall wood density over all the clonal material.

Table VI. Analysis of variance $(F$-value and correspondent $p, p<$ 0.05 in bold) for clone and competition index for tree diameter, height and average ring width (RW).

\begin{tabular}{lccccc}
\hline \multirow{2}{*}{ Trait } & \multicolumn{3}{c}{ Clone } & \multicolumn{2}{c}{ Competition Index } \\
\cline { 2 - 6 } & MSE & $F$-ratio & $P$-value & $F$-ratio & $P$-value \\
\hline Diameter & 7.34 & 1.72 & $\mathbf{0 . 0 5}$ & 35.70 & $\mathbf{0 . 0 0}$ \\
Height & 3.52 & 2.01 & $\mathbf{0 . 0 2}$ & 74.52 & $\mathbf{0 . 0 0}$ \\
RW & 0.22 & 2.25 & $\mathbf{0 . 0 1}$ & 29.62 & $\mathbf{0 . 0 0}$ \\
\hline
\end{tabular}

\section{DISCUSSION AND CONCLUSIONS}

In the present work, the phenotypic variation for wood density traits and fibre properties was considerably lower than for growth or yield traits, following similar patterns of previous investigations (e.g. Zubizarreta Gerendiain et al., 2008). Such findings would imply significantly stronger genetic control of fibre properties and wood density traits compared to growth and yield traits, which are more affected by environmental conditions, and therefore also from competition for resources (e.g. Zobel and Jett, 1995). Despite this, one of the largest concerns in tree breeding for Norway spruce is the negative correlation between the growth rate of trees and wood density (e.g. Mäkinen, 2002a; Zubizarreta Gerendiain et al., 2007). Nonetheless, even if this was the general pattern also in our work, we found that some Norway spruce clones showed both higher average diameter and overall wood density than the average for all the clones. On the other hand, we also observed clones which concurrently had both low growth rate and overall wood density; possibly indicating a short growing period. Thus, our findings could be seen as an opportunity to obtain, by proper selection, clones with the desired properties, and at the same time discard the undesired ones.

We observed differences between clones for all yield traits, wood density traits and fibre properties (excluding coarseness). In this sense, Steffenrem et al. (2007) found differences among Norway spruce families for height and wood density, but not for diameter and RW. Concerning origins, we did not find significant differences, on average, for growth and yield traits (excluding LWW\%) between Finnish, Russian and provenance-hybrids, while in some wood density traits and fibre properties (WD, EWD and FWT) we observed differences. As a comparison, Fottland and Skrøppa (1989) found differences in height growth of various Norway spruce provenances, while Bujold et al. (1996) also found differences in height, but not in diameter or stem volume. Generally, the possible reason for larger differences between clones compared to those for origins could be that the differences related to the provenances are relatively small compared to individual tree or family variation (Zobel and Jett, 1995). In this sense, the larger differences between clones than between origins could suggest that selection for a desired property trait or a combination of traits would be more beneficial based on a specific clone instead of an entire provenance.

In some previous studies, southern provenances of Norway spruce, as well as other conifers, grew better (e.g. tree height) due to their longer growing period than the local provenances when planted in more northern locations, indicating that the growth rhythm (initialisation and cessation of tree growth) may be closely determined by the provenances (e.g. Skrøppa and Magnussen, 1993; Jayawickrama, 1998). In our study, we could not find such generalisations related to yield traits since, for example, the tallest clones were some Finnish clones $(\mathrm{F} \times \mathrm{F})$, while the shortest ones were a German-Finnish clone $(F \times G)$ and a Finnish clone $(F \times F)$.

When analysing the wood density traits in greater detail, we observed differences among the individual clones for all traits, as was also observed in previous investigations (e.g. Zubizarreta Gerendiain et al., 2007). Furthermore, the clones originating from the most northern areas, such as Russia, Finland or the Estonia-Finland cross, had, on average, the highest overall wood density. Our findings are in line with previous studies, where the northern provenances showed higher wood density than their southern counterparts (Persson and Persson, 1997; Skrøppa et al., 1999). This has been explained by the fact that northern provenances finish the height growth earlier during the growing season, and consequently they also finish earlywood formation earlier in the growing season, thus, 
Table VII. Phenotypic correlations between yield, growth, wood density traits and fibre properties as an average for all the clones. Statistically significant correlations $(p<0.05)$ shown in bold.

\begin{tabular}{|c|c|c|c|c|c|c|c|c|c|c|c|c|c|}
\hline Traits* & $H$ & $V$ & EWW & LWW & RW & EWD & LWD & WD & LWW\% & FL & FW & $\mathrm{C}$ & FWT \\
\hline $\mathrm{D}$ & 0.85 & 0.96 & 0.85 & 0.69 & 0.86 & -0.47 & -0.16 & -0.54 & -0.58 & 0.35 & 0.62 & 0.39 & 0.11 \\
\hline $\mathrm{H}$ & & 0.81 & 0.65 & 0.54 & 0.67 & -0.36 & 0.08 & -0.39 & -0.53 & 0.53 & 0.70 & 0.58 & 0.36 \\
\hline V & & & 0.81 & 0.64 & 0.83 & -0.43 & -0.21 & -0.52 & -0.53 & 0.25 & 0.55 & 0.33 & 0.07 \\
\hline EWW & & & & 0.64 & 0.99 & -0.55 & -0.33 & -0.71 & -0.76 & 0.13 & 0.46 & 0.15 & -0.15 \\
\hline LWW & & & & & 0.74 & -0.31 & -0.18 & -0.24 & -0.07 & 0.19 & 0.38 & 0.23 & 0.06 \\
\hline RW & & & & & & -0.54 & -0.32 & -0.67 & -0.68 & 0.15 & 0.47 & 0.17 & -0.12 \\
\hline EWD & & & & & & & 0.53 & 0.89 & 0.48 & -0.25 & -0.12 & -0.01 & 0.09 \\
\hline LWD & & & & & & & & 0.69 & 0.22 & 0.43 & 0.32 & 0.53 & 0.60 \\
\hline WD & & & & & & & & & 0.75 & -0.06 & -0.12 & 0.12 & 0.31 \\
\hline LWW\% & & & & & & & & & & -0.13 & -0.35 & -0.10 & 0.17 \\
\hline FL & & & & & & & & & & & 0.72 & 0.79 & 0.70 \\
\hline FW & & & & & & & & & & & & 0.87 & 0.55 \\
\hline C & & & & & & & & & & & & & 0.89 \\
\hline
\end{tabular}

* See Table II for the explanation of the traits.

having a longer period for latewood formation, which has higher density (Jayawickrama et al., 1997, 1998). On the contrary, Wilhelmsson et al. (2002) showed, in Norway spruce grown in Sweden, that north-Swedish provenances had, on average, lower overall wood density due to the short growing season in the north, which results in a shorter period for latewood formation.

In previous studies on the effects of provenance, carried out in southern Norway (e.g. Skrøppa et al., 1999) or Sweden (e.g. Persson and Persson, 1997) the provenance transfer from south to north was recommended in order to match phonological behaviour in spring and autumn. However, the lack of proper adaptability of the southern most origins to Finnish climatic conditions could be one reason for the different results we observed, since the Finnish ones showed the highest growth. Thus, based on our investigation, the transfer of our study material representing the southern most provenances to more northern locations could not be recommended. Nonetheless, unfortunately, the lack of detailed information regarding the latitude and altitude of the parent trees of the foreign clones or provenance-hybrids made it impossible to evaluate, in greater detail, the reasons behind the different responses observed between clones at this stage. In this sense, more detailed information on the provenances of the clones would be needed (e.g. temperature sum for geographical origins of parent trees) to make more accurate conclusions.

To study the possible effects of neighbour tree competition, we also applied a competition index (CI) formulated by Schütz (1989), which is based on tree height, crown width and distance between target tree and its neighbours. The results showed that the CI could help to explain part of the variability of yield traits. Similarly, in previous investigations of such tree species as white fir (Abies concolor), ponderosa pine and loblolly pine, significant improvements in the prediction of tree growth was achieved by taking into account the effects of the competition (Biging and Dobbertin, 1992; Jayawickrama, 1998).

Nonetheless, in future studies more attention should be paid to enhance our understanding on the effects of competition, on growth, yield and wood property traits of different genetic entries, especially after canopy closure (Biging and Dobbertin, 1992). Similarly, annual variability of climatic conditions should also be considered side-by-side with annual growth and wood properties to draw more detailed conclusions on the suitability of different genetic entries as regeneration material for Finnish conditions under changing climate.

Acknowledgements: This work was related to the Centre of Excellence for Boreal Forest Management Research (2007-2009) lead by Prof. Seppo Kellomäki. The support by the Graduate School for Forest Sciences is gratefully acknowledged. The finnish forest research institute (FFRI) is thanked for providing the material and Dr. Antti Kilpeläinen, Ms. Marja Kuskelin and Mr. Jarmo Pennala from University of Joensuu and Ms. Pirita Lohela and trainees from FFRI for helping in field work. Prof. José Tomé from Instituto Superior de Agronomía (Lisbon) is thanked for the advice for the statistical analyses. Mr. Jarmo Pennala and Ms. Maini Mononen (University of Joensuu) are also thanked for laboratory work and Mr. David Gritten for the English revision.

\section{REFERENCES}

Bergsten U., Lindeberg J., Rindby A., and Evans R., 2001. Batch measurements of wood density on intact or prepared drill cores using X-ray microdensitometry. Wood Sci. Technol. 35: 435-452.

Biging G.S. and Dobbertin M., 1992. A comparison of distancedependent competition measures for height and basal area growth of individual conifer trees. For. Sci. 38: 695-720

Bujold S.J., Simpson J.D., Beukeveld J.H.J., and Schneider, M.H., 1996. Relative density and growth of eleven Norway spruce provenances in Central New Brunswick. North J. Appl. For. 13(3): 124-128.

Cajander A.K., 1926. The Theory of Forests Types, Acta For. Fenn. 29: $1-108$.

Ekensted F., Grahn T., Hedenberg Ö., Lundqvist S.-O., Arlinger J., and Wilhelmsson L., 2003. Variations in fiber dimensions of Norway spruce and Scots pine. Swedish Pulp and Paper Research Institute, Stockholm, Sweden STFI Report, PUB13, 36 p.

Fottland H. and Skrøppa T., 1989. The IUFRO 1964/68 provenance experiment with Norway spruce (Picea abies) in Norway: variation in mortality and height growth. Meddelelser fra Norsk institutt for skogforskning 43.1: $30 \mathrm{p}$. 
Hegyi F., 1974. A simulation model for managing jack-pine stands. In: Fries, G. (Ed.), Growth Models for Tree and Stand Simulation. Royal College of Forestry, Stockholm, Sweden. Res. Note 30, pp. 74-90.

Jayawickrama K.J.S., McKeand S.E., Jett J.B., and Wheeler E.A., 1997. Date of earlywood-latewood transition in provenances and families of loblolly pine, and its relationship to growth phenology and juvenile wood specific gravity. Can. J. For. Res. 27: 1245-1253.

Jayawickrama K.J.S., McKeand S.E., and Jett J.B., 1998. Phenological variation in height and diameter growth in provenances and families of loblolly pine. New For. 16: 11-25.

Laasasenaho J., 1982. Taper curve and volume functions for pine, spruce and birch. Commun. Inst. For. Fenn. 108: 1-74.

Mäkinen H., 1997. Possibilities of competition indices to describe competitive differences between Scots pine families. Silva Fenn. 31: $43-52$.

Mäkinen H., Saranpää P., and Linder S., 2002a. Wood-density variation of Norway spruce in relation to nutrient optimization and fibre dimensions. Can. J. For. Res. 32: 185-194.

Mäkinen H., Saranpää P., and Linder S., 2002b. Effect of growth rate on fibre characteristics in Norway spruce (Picea abies (L.) Karst.). Holzforschung 56: 449-460.

Persson B. and Persson A., 1997. Variation in stem properties in a IUFRO 1964/1968 Picea abies provenance experiment in Southern Sweden. Silvae Genet. 2-3: 94-101.

Pukkala T., 1989. Methods to describe the competition process in a tree stand. Scand. J. For. Res. 4: 187-202.

Schütz J.P., 1989. Zum Problem der Konkurrenz in Mischbeständen. Schweiz. Z. Forstwes. 140: 1069-1083.

Skrøppa T. and Magnussen S., 1993. Provenance variation in shoot growth components of Norway spruce. Silvae Genet. 42: 111-119.

Skrøppa T., Hylen G., and Dietrichson J., 1999. Relationships between wood density components and juvenile height growth and growth rhythm traits for Norway spruce provenances and families. Silvae Genet. 48: 235-239.
Ståhl E. 1998. Changes in wood and stem properties of Pinus sylvestris caused by provenance transfer. Silva Fenn. 32: 163-172.

Steffenrem A., Saranpää P., Lundqvist S.O., and SKroppa T. 2007. Variation in wood properties among five full-sib families of Norway spruce (Picea abies) Ann. For. Sci. 64: 799-806.

Tomé M. and Burkhart H.E., 1989. Distance-dependent competition measures for predicting growth of individual trees. For. Sci. 35: 816-831.

Tyrväinen J., 1995. Wood and fiber properties of Norway spruce and its suitability for thermomechanical pulping. Acta For. Fenn. 249: 1155.

Wang T., Aitken S. N., Rozenberg P., and Millie F., 2000. Selection for improved growth and wood density in Lodgepole pine: effects on radial patterns of wood variation. Wood Sci. Technol. 32: 391-403.

Wilhelmsson L., Arlinger J., Spångberg K., Lundqvist S-O., Grahn T., Hedenberg Ö., and Olsson L., 2002. Models for predicting wood properties in stems of Picea abies and Pinus sylvestris in Sweden. Scand. J. For. Res. 17: 330-350.

Zhang S.Y. and Morgenstern E.K., 1995. Genetic variation and inheritance of wood density in black spruce (Picea mariana) and its relationship with growth: implications for tree breeding. Wood Sci. Technol. 30: 63-75.

Zobel B.J. and van Buijtenen J.P., 1989. Wood variation: Its causes and control. Springer-Verlag, Berlin, Germany, 363 p.

Zobel B.J. and Jett J.B., 1995. Genetics of wood production, SpringerVerlag, Berlin.

Zubizarreta Gerendiain A., Peltola H., Pulkkinen P., Jaatinen R., Pappinen A., and Kellomäki S., 2007. Differences in growth and wood property traits in cloned Norway spruce (Picea abies). Can. J. For. Res. 37: 2600-2611.

Zubizarreta Gerendiain A., Peltola H., Pulkkinen P., Jaatinen R., and Pappinen A., 2008. Differences in fibre properties in cloned Norway spruce (Picea abies). Can. J. For. Res. 38: 1071-1082. 